To appear in Waves in Random Media - a topical issue on "Disordered Electronic Systems"

\title{
Orbital Magnetism in Disordered Mesoscopic Metals
}

\author{
B. Shapiro \\ Department of Physics, Technion, Haifa 32000
}

\begin{abstract}
The theory of orbital magnetism in disordered metals is reviewed, and extended to include a broad range of temperatures and fields. Sample-tosample fluctuations in the orbital magnetic susceptibility are studied. In a given sample these fluctuations manifest themselves in aperiodic samplespecific oscillations of susceptibility and magnetization, when the strength of the magnetic field is changed.
\end{abstract}

\section{Introduction}

A degenerate electron gas in a macroscopic metal exhibits weak orbital magnetism (the Landau diamagnetism) [1]. There are two factors that can significantly enhance the orbital magnetic response: finite system size and electron-electron interactions. This paper is devoted primarily to reviewing and extending recent work on the finite size (i.e. mesoscopic) effects on orbital magnetism of disordered metals (some of the results obtained prior 
to 1993 have been reviewed in [2]). The effect of interactions will be briefly addressed at the end of the paper.

In a weakly disordered metal the elastic mean free path $\ell$ is much larger than the inverse Fermi number $k_{F}^{-1}$ but much smaller than the sample size L. Under these conditions electrons propagate by a diffusion process, with a diffusion coefficient $D=v_{F} \ell / d$, where $v_{F}$ is the Fermi velocity and $d$ is the sample dimensionality. In addition to the three length scales $\left(k_{F}^{-1}, \ell, L\right)$ which characterize the sample, there are two more lengths, $L_{T}=(\hbar D / T)^{\frac{1}{2}}$ and $L_{B}=(\hbar c / e B)^{\frac{1}{2}}$, which specify the temperature $T$ and the external magnetic field $B$. We assume that the inelastic scattering (or the phase breaking) length is larger than $L_{T}$ and, thus, irrelevant for our purpose.

Whether a sample of a given size $L$ can be considered as macroscopic, depends on the temperature. If $T$ is smaller than the Thouless energy $E_{c} \simeq$ $\hbar D / L^{2}$ (i.e. $L_{T}>L$ ), the sample forms a single coherent unit and can exhibit large mesoscopic effects. The orbital magnetic susceptibility $\chi$ fluctuates from sample to sample and the typical fluctuation, $\left\langle\Delta \chi^{2}\right\rangle^{\frac{1}{2}}$, can exceed the average value $\langle\chi\rangle$ by a large factor (angular brackets denote averaging over an ensemble of macroscopically identical samples). Mesoscopic effects in orbital magnetism are not restricted to $T<E_{c}$ but persist to much higher temperatures, although their magnitude gradually decreases [3-6]. A detailed study of $\left\langle\Delta \chi^{2}\right\rangle^{\frac{1}{2}}$, in the temperature regime $E_{c} \ll T \ll \hbar v_{F} / \ell$, was made in $[5,6]$. Some of these results will be re-derived below. The present treatment, however, allows us to consider a much broader range of temperatures and 
fields. In particular, temperatures larger than $\hbar v_{F} / \ell$ (i.e. $L_{T}<\ell$ ) and fields stronger than $\hbar c \ell^{2} / e\left(\right.$ i.e. $\left.L_{B}<\ell\right)$ will be discussed.

In Sec. 2 we explain the phenomenon of mesoscopic orbital magnetism using an example of a clean chaotic cavity. A clear understanding of this simple system is helpful for the qualitative considerations of Sec. 3. A quan-

titative theory for $\left\langle\Delta \chi^{2}\right\rangle^{\frac{1}{2}}$ of a disordered metal is set up in Sec. 4, and its consequences are examined in Sec. 5, for various temperatures and fields. Sec. 6 is devoted to a brief discussion of the interaction effects. Conclusions are summarized in Sec. 7 .

\section{Chaotic cavities}

Before turning to disordered metals, let us briefly discuss the simpler case of a clean chaotic cavity. We follow below the presentation in [2]. (Similar considerations for persistent currents appear in $[7,8]$.) The two relevant energies are the level spacing $\Delta$ and the "inverse time of flight" across the cavity, $\Gamma \simeq \hbar v_{F} / L$. The mesoscopic regime corresponds to the temperature range $\Delta \ll T \lesssim \Gamma$. In this regime the thermodynamics of the system is determined by the density of states $\rho_{\Gamma}(\epsilon)$, smoothed over an interval of order $\Gamma$ near energy $\epsilon$. The main point is that $\rho_{\Gamma}$ contains a small oscillatory component, $\delta \rho_{\Gamma}$, which is due to the shortest periodic orbit $[9]$

$$
\delta \rho_{\Gamma}(\epsilon, B) \simeq \frac{1}{\Gamma} \cos (k L+\gamma) \cos \left(\frac{2 \pi B L^{2}}{\phi_{0}}\right) .
$$

where $k=\hbar^{-1} \sqrt{2 m \epsilon}, \phi_{0}=2 \pi \hbar c / e$, and the magnetic field $B$ is assumed to be weak, so that the cyclotron radius $R_{c}=v_{F} m c / e B$ is larger than the size 
$L$ of the cavity. The oscillatory term in the density of states contributes a small oscillatory correction $\delta \Omega$ to the thermodynamic potential:

$$
\delta \Omega=-T \int d \epsilon \delta \rho_{\Gamma}(\epsilon) \ln \left[1+\exp \left(\frac{\mu-\epsilon}{T}\right)\right],
$$

which, after integrating by parts twice and keeping the leading term in the large parameter $k_{F} L$, gives

$$
\delta \Omega \simeq \Gamma \cos \left(k_{F} L+\gamma\right) \cos \left(\frac{2 \pi B L^{2}}{\phi_{0}}\right) .
$$

It is this correction that, due to its sensitivity to the magnetic field, dominates the differential magnetic susceptibility

$$
\chi=-L^{-d} \frac{\partial^{2} \Omega}{\partial B^{2}} \simeq \chi_{0}\left(k_{F} L\right)^{3-d} \cos \left(k_{F} L+\gamma\right) \cos \left(\frac{2 \pi B L^{2}}{\phi_{0}}\right),
$$

where $\chi_{0} \simeq e^{2} k_{F}^{d-2} / m c^{2}$ is the absolute value of the Landau susceptibility, in $d$ dimensions.

Thus, the orbital susceptibility of a two-dimensional electron gas confined to a chaotic cavity (a quantum dot) is enhanced by a factor $k_{F} L$, as compared to $\chi_{0}$. Detailed semiclassical computations of the orbital magnetic susceptibility for a generic chaotic system have been performed by Agam [10], and for a "stadium billiard" by Raveh [6]. Similar semiclassical considerations apply also to integrable systems [11-13] where, due to existence of families of periodic orbits, the enhancement is even larger. In all clean systems mesoscopic effects rapidly disappear when temperature is raised above $\Gamma$, i.e. for $T \gg \Gamma$ the system becomes macroscopic and its orbital susceptibility assumes the Landau value. This is in contrast to the situation in disordered metals, where 
the effect decays with temperature rather gradually.

\section{Disordered Metals: Qualitative Considerations}

The following qualitative discussion is confined to two-dimensional samples. In a disordered metal electrons propagate diffusively, so that the energy $\Gamma$ of the previous section should be replaced by the Thouless energy $E_{c}=\hbar D / L^{2}$. For $T \lesssim E_{c}$, a change $\delta B \simeq \phi_{0} / L^{2}$ in a magnetic field produces a change $\delta \Omega \simeq E_{c}$ in the thermodynamic potential. The typical magnetic susceptibility $\chi_{\text {typ }}$ of a given sample can have either sign, and its magnitude is of order $\delta \Omega / L^{2} \delta B^{2} \simeq \chi_{0} k_{F} \ell$.

The large sample-to-sample fluctuations cancel out, to a large extent, if one averages over many samples, with different impurity arrangements. The resulting average value $\langle\chi\rangle$ is much smaller than $\chi_{\text {typ }}$ and depends on whether the samples are in contact with particle reservoir (grand canonical system) or are isolated (a canonical one). In the former case $\langle\chi\rangle \approx-\chi_{0}=-e^{2} / 12 \pi m c^{2}$, whereas in the latter there is an additional paramagnetic contribution which, for $T \ll E_{c}$, is the dominant one [4,14]. We will concentrate on the typical susceptibility, or, more precisely, on its fluctuating part $\left\langle\Delta \chi^{2}\right\rangle^{\frac{1}{2}} \equiv \delta \chi$. This quantity is insensitive to the type of thermodynamic ensemble.

Thus, for $T \lesssim E_{c}, \delta \chi \simeq \chi_{0} k_{F} \ell$. Let us see how $\delta \chi$ changes when $T$ is raised above $E_{c}$. For $E_{c} \ll T \ll\left(\hbar v_{F} / \ell\right) \equiv \hbar / \tau$ (i.e. $\left.\ell \ll L_{T} \ll L\right)$, one can view the sample as made up of $\left(L / L_{T}\right)^{2}$ boxes, of size $L_{T}$ each. The above estimate is applicable for each box separately, i.e. $\delta \chi_{\text {Box }} \simeq \chi_{0} k_{F} \ell$ and the fluctuating part of the magnetic moment of a box is $\delta M_{\mathrm{Box}} \simeq \chi_{0} k_{F} \ell B L_{T}^{2}$. Summing 
over all boxes, with random signs, one obtains the typical fluctuation of the magnetic moment of the entire sample, $\delta M \simeq \chi_{0} k_{F} \ell B L_{T}^{2}\left(L / L_{T}\right)$, i.e. $\delta \chi / \chi_{0} \simeq k_{F} \ell\left(L_{T} / L\right) \sim T^{-\frac{1}{2}}$. This ratio is smaller than $k_{F} \ell$ but can be larger than unity. Even if this relative fluctuation is small, it can still be of interest because of its non-trivial temperature and field dependence (strong field effects will be discussed later).

The picture changes, if the temperature is raised further and becomes larger than $\hbar / \tau$ (i.e. $L_{T}<\ell$ ). The relevant trajectories, which dominate the magnetic response, are now of ballistic (rather than diffusive) nature, i.e. shorter than $\ell$. Only short trajectories, of an area $S \lesssim\left(\hbar v_{F} / T\right)^{2} \equiv \ell_{T}^{2}$, can efficiently respond to the magnetic flux. Let us denote by $W_{T}$ the probability for having this kind of a trajectory, in a "box" of size $\ell_{T}$. The probability $P(S) d S$ for a closed ballistic trajectory, of an area between $S$ and $S+d S$, was estimated in [15] as $P(S) d S \simeq \ell^{-3} \sqrt{S} d S$, so that $W_{T} \simeq\left(\ell_{T} / \ell\right)^{3}$. The fluctuating part of the susceptibility $\delta \chi$ of the sample is estimated in a way similar to that used above for the diffusive regime: The sample is partitioned into $\left(L / \ell_{T}\right)^{2}$ boxes; a box contains, with probability $W_{T}$, a ballistic trajectory of an area of order $\ell_{T}^{2}$ which contributes a magnetic moment $\left|\delta M_{\mathrm{Box}}\right| \simeq$ $\chi_{0} k_{F} \ell_{T} B \ell_{T}^{2}$ (the factor $k_{F} \ell_{T}$ is the enhancement factor discussed in Sec. 2). Adding up the (random) contributions of all boxes, and remembering that only a fraction $W_{T}$ of all boxes does carry magnetic moments, one obtains $\delta M \simeq\left|\delta M_{\mathrm{Box}}\right|\left(W_{T} L^{2} / \ell_{T}^{2}\right)^{\frac{1}{2}}$, i.e. $\delta \chi \simeq \chi_{0} k_{F} \ell(\ell / L)\left(\ell_{T} / \ell\right)^{\frac{7}{2}}$. Thus, the $T^{-\frac{1}{2}}$ dependence of the diffusion regime $\left(E_{c}<T<\hbar / \tau\right)$ crosses over to a $T^{-\frac{7}{2}}$ dependence in the ballistic regime $(T>\hbar / \tau)$. 
The arguments so far were restricted to weak magnetic fields, i.e. to the regime of linear response. The region of validity of linear response depends, of course, on temperature. For instance, in the diffusion regime linear response requires $L_{B}>L_{T}$, i.e. $\hbar \omega_{c}<T / k_{F} \ell$, where $\omega_{c}$ is the cyclotron frequency. For stronger fields various non-linear effects in susceptibility and magnetization show up. In particular, for $L_{B}<L_{T}$, one may expect aperiodic, samplespecific fluctuations in magnetization [6]: every time when the magnetic field acquires an increment $\Delta B \simeq \phi_{0} / L_{T}^{2}$, an effectively "new sample" is created and, thus, the differential susceptibility changes by an amount of order $\pm \chi_{0} k_{F} \ell\left(L_{T} / L\right)$. These sample-specific fluctuations resemble the universal conductance fluctuations (see [16] for an extensive discussion), although the relative fluctuation in susceptibility is much larger than in the conductance.

\section{Disordered Metals: Theory}

The grand potential, for a specific sample, is

$$
\Omega=V_{0} \int d \epsilon \nu(\epsilon, B) F_{T}(\epsilon-\mu),
$$

where $V_{0}=L^{d}$ is the sample volume, $\nu(\epsilon, B)$ is the density of states at field $B$, per unit volume, and

$$
F_{T}(\epsilon-\mu)=-T \ln \left[1+\exp \left(-\frac{\epsilon-\mu}{T}\right)\right] .
$$

The sample magnetization is $M=-V_{0}^{-1}(\partial \Omega / \partial B)_{\mu, V, T}$ and its differential susceptibility is $\chi=\partial M / \partial B$. The variance, $\left\langle\Delta \chi^{2}\right\rangle$, in the ensemble of random samples is therefore

$$
\left\langle\Delta \chi^{2}\right\rangle=\lim _{B_{2} \rightarrow B} \lim _{B_{1} \rightarrow B} \frac{\partial^{4}}{\partial B_{1}^{2} \partial B_{2}^{2}} \iint d \epsilon_{1} d \epsilon_{2} F_{T}\left(\epsilon_{1}-\mu\right) F_{T}\left(\epsilon_{2}-\mu\right) K\left(\epsilon_{1}, \epsilon_{2} ; B_{1}, B_{2}\right),
$$


where $K\left(\epsilon_{1}, \epsilon_{2} ; B_{1}, B_{2}\right)=\left\langle\Delta \nu\left(\epsilon_{1}, B_{1}\right) \Delta \nu\left(\epsilon_{2}, B_{2}\right)\right\rangle$ is the correlation function for the density of states, at two different energies and for two close values of the magnetic field.

The density of states correlation function for diffusive metals was first considered by Altshuler and Shklovskii [17]. In the context of orbital magnetism it has been studied by a number of authors [4-6,14,18]. The computations are based on writing the density of states in terms of retarded and advanced Green's functions:

$$
\nu(\epsilon, B)=-s \frac{i}{2 \pi V} \operatorname{Tr}\left[G_{R}(\epsilon, B)-G_{A}(\epsilon, B)\right]
$$

where the factor $s=2$ accounts for spin degeneracy. In what follows it is useful to use the approach of Altland and Gefen [18] and to write

$$
\begin{aligned}
G_{R, A}(\epsilon, B) & =\frac{\partial}{\partial \epsilon} \ln \left(\epsilon \pm i \eta-H_{0}-V\right)= \\
& =\frac{\partial}{\partial \epsilon}\left[\ln \left(\epsilon \pm i \eta-H_{0}\right)+\sum_{n=1}^{\infty} \frac{1}{n}\left(G_{R, A}^{(0)} V\right)^{n}\right]
\end{aligned}
$$

where $H_{0}=(1 / 2 m)\left(p-\frac{e}{c} A\right)^{2}$ is the unperturbed Hamiltonian, $G_{R, A}^{(0)}$ are the corresponding Green's functions and $V$ is the random potential. Eq.(9) is written in an operator form, rather than in the coordinate representation. Averaging of a product $G_{R}\left(\epsilon_{1}, B_{1}\right) G_{A}\left(\epsilon_{2}, B_{2}\right)$ results in the usual "diffuson" and "cooperon" series. The contribution of the $n$-th term of the cooperon (diffuson) series to the correlation function $K\left(\epsilon_{1}, \epsilon_{2} ; B_{1}, B_{2}\right)$ is:

$$
K_{ \pm}^{(n)}=\frac{s^{2}}{2 \pi^{2} V_{0}^{2}} \frac{\partial^{2}}{\partial \epsilon_{1} \partial \epsilon_{2}}\left[\frac{1}{n} \operatorname{Re} \operatorname{Tr} P_{ \pm}^{n}\left(\epsilon_{1}-\epsilon_{2}, B_{ \pm}\right)\right]
$$


where sign $+(-)$ corresponds to cooperon (diffuson), and $B_{ \pm}=B_{1} \pm B_{2}$. The operator $P_{+}$, in coordinate representation, is written as

$$
P_{+}\left(\vec{r}, \vec{r}^{\prime} ; \epsilon_{1}-\epsilon_{2}, B_{+}\right)=\frac{\hbar}{2 \pi \nu_{0} \tau}\left\langle G_{R}\left(\vec{r}, \vec{r}^{\prime} ; \epsilon_{1}, B_{1}\right)\right\rangle\left\langle G_{A}\left(\vec{r}, \vec{r}^{\prime} ; \epsilon_{2}, B_{2}\right)\right\rangle
$$

where $\nu_{0}$ is the free electron density of states (per spin), for an infinite system at $B=0$. The expression for $P_{-}\left(\vec{r}, \vec{r}^{\prime} ; \epsilon_{1}-\epsilon_{2}, B_{-}\right)$is obtained from Eq. (11) by interchanging $\vec{r}$ and $\vec{r}^{\prime}$, in the advanced Green's function. For classically weak magnetic fields, i.e. when $\omega_{c} \tau \ll 1$,

$$
\left\langle G_{R, A}\left(\vec{r}, \vec{r}^{\prime} ; \epsilon, B\right)\right\rangle=\left\langle G_{R, A}\left(\vec{r}, \vec{r}^{\prime} ; \epsilon, B=0\right)\right\rangle \exp \left(\frac{i e}{\hbar} \int_{\vec{r}}^{\vec{r}^{\prime}} \vec{A} \cdot d \vec{\ell}\right)
$$

where the integration is along a straight line connecting $\vec{r}$ to $\vec{r}^{\prime}$.

To obtain the full function $K\left(\epsilon_{1}, \epsilon_{2} ; B_{1}, B_{2}\right)$ one has to sum $K_{ \pm}^{(n)}$ over $n$. Since, eventually, derivatives with respect to $B_{1}, B_{2}$ are to be taken [Eq. (7)], one is interested only in the $B$-dependent part of $K$. The (single) term with $n=1$ and the two terms with $n=2$ (one for diffuson, one for cooperon) do not depend on $B$. This happens because the $B$-dependent phase factor drops out in a diagonal term $\left\langle G_{R}(\vec{r}, \vec{r})\right\rangle$, as well as in the product $\left\langle G_{R}\left(\vec{r}, \vec{r}^{\prime}\right)\right\rangle\left\langle G_{R}\left(\vec{r}^{\prime}, \vec{r}\right)\right\rangle$ (and similarly for the advanced Green's functions). Thus, the final expression for the $B$-dependent part, $\Delta K$, of $K$ is:

$$
\begin{aligned}
& \Delta K\left(\epsilon_{1}, \epsilon_{2} ;\right.\left.B_{1}, B_{2}\right)=\frac{s^{2}}{2 \pi^{2} V_{0}^{2}} \frac{\partial^{2}}{\partial \epsilon_{1} \partial \epsilon_{2}} R e \sum_{n=3}^{\infty} \frac{1}{n} \sum_{\alpha}\left(\lambda_{\alpha}^{n}\left(\epsilon_{1}-\epsilon_{2}, B_{+}\right)+\right. \\
&\left.+\lambda_{\alpha}^{n}\left(\epsilon_{1}-\epsilon_{2}, B_{-}\right)\right)
\end{aligned}
$$

where $\lambda_{\alpha}\left(\epsilon_{1}-\epsilon_{2}, B_{ \pm}\right)$is the $\alpha$-th eigenvalue of the operators $P_{ \pm}$.

Eq. (13), supplemented by the definition (11) of the operators $P_{ \pm}$, forms the basis for a quantitative treatment of orbital magnetism in disordered 
metals, in a broad range of temperatures and fields. The treatment is not restricted [18] to the standard diffusion approximation for cooperon and diffuson. The situation is the same as in the treatment of the weak localization correction for the conductivity. This correction is expressed in terms of the eigenvalues of the operator $P_{+}$and, in order to obtain reasonably accurate quantitative results in a broad range of magnetic fields, one has to evaluate these eigenvalues beyond the diffusion approximation [15, 19-21].

Physical quantities of interest can be expressed in terms of $\Delta K$, given in Eq. (13). Along with the variance

$$
\left\langle\Delta \chi^{2}\right\rangle=\lim _{\substack{B_{1} \rightarrow B \\ B_{2} \rightarrow B}} \frac{\partial^{4}}{\partial B_{1}^{2} \partial B_{2}^{2}} \iint d \epsilon_{1} d \epsilon_{2} F_{T}\left(\epsilon_{1}-\mu\right) F_{T}\left(\epsilon_{2}-\mu\right) \Delta K\left(\epsilon_{1}, \epsilon_{2} ; B_{1}, B_{2}\right)
$$

one can consider various correlation functions, such as $\langle\Delta \chi(B) \Delta \chi(B+\Delta B)\rangle$ or $\langle\Delta \chi(T) \Delta \chi(T+\Delta T)\rangle$. The first function is defined by Eq. (14) with $B_{1} \rightarrow B, B_{2} \rightarrow B+\Delta B$. To obtain the second function one has to replace one of the $F_{T}$-factors in Eq. (14) by $F_{T+\Delta T}$. Other correlation functions, for susceptibility or magnetization, can be defined in a similar way.

Integration in Eq. (14) can be carried out. After integration by parts, the derivatives contained in $\Delta K$ act on the $F_{T}$-factors, giving

$$
\frac{d}{d \epsilon} F_{T}(\epsilon-\mu)=\left[\exp \left(\frac{\epsilon-\mu}{T}\right)+1\right]^{-1} \equiv f_{T}(\epsilon-\mu)
$$

The subsequent integration reduces to the Matsubara sums over the poles of $f_{T}(\epsilon-\mu)$. This introduces a factor $(2 \pi T)^{2}$ and changes the argument $\epsilon_{1}-\epsilon_{2}$ of the functions under the integral to $\epsilon_{p}+\epsilon_{q}$ where $\epsilon_{p}=i \pi T(2 p+1)$, $\epsilon_{q}=i \pi T(2 q+1)$ and $p, q=0,1, \ldots$ The final result is (the double sum 
over fermionic frequencies can be replaced by a single sum over a bosonic frequency):

$$
\begin{gathered}
\left\langle\Delta \chi^{2}\right\rangle=\frac{2 s^{2} T^{2}}{V_{o}^{2}} \lim _{\substack{B_{1} \rightarrow B \\
B_{2} \rightarrow B}} \frac{\partial^{4}}{\partial B_{1}^{2} \partial B_{2}^{2}} \operatorname{Re} \sum_{n=3}^{\infty} \frac{1}{n} \sum_{p, q=0}^{\infty} \sum_{\alpha}\left[\lambda_{\alpha}^{n}\left(\epsilon_{p}+\epsilon_{q}, B_{+}\right)+\right. \\
\left.+\lambda_{\alpha}^{n}\left(\epsilon_{p}+\epsilon_{q}, B_{-}\right)\right]
\end{gathered}
$$

In the next Section we study $\left\langle\Delta \chi^{2}\right\rangle$, for various temperatures and fields, and briefly discuss some correlation functions.

\section{Results and Discussion}

One should distinguish between weak magnetic fields, $L_{B}>L_{T}$ (linear response) and stronger fields, when the dependence of magnetization on $B$ becomes non-linear. Note that $L_{B}>L_{T}$ is a sufficient condition for linear response, i.e. there is no requirement that $L_{B}$ should be larger than the sample size $L$ (recall that we are interested in the "high temperature" case, $L_{T}<L$ ). In this respect the situation is like in the thermodynamic perturbation theory [1], where finite temperature stabilizes the perturbative treatment and enlarges its range of validity. Thus, while computing the eigenvalues of the operators $P_{ \pm}$, we can assume $L_{B}<L$. Moreover, it is sufficient to find only the eigenvalues of $P_{+}$. This is because the function $P_{-}$differs from $P_{+}$only by its argument, containing $\left(B_{1}-B_{2}\right)$ instead of $\left(B_{1}+B_{2}\right)$. In particular,

since in the definition of $\left\langle\Delta \chi^{2}\right\rangle$ the difference $\left(B_{1}-B_{2}\right)$ is infinitesimal, the contribution of $P_{-}$to $\left\langle\Delta \chi^{2}\right\rangle$ is the same as of $P_{+}$in the limit of small $B$.

Eigenvalues of the operator $P_{+}$are well known, in connection with the weak localization problem [20] (a closely related operator appears also in the 
theory of superconductivity $[22,23])$. For the two-dimensional case, which is under consideration here, the $N$-th eigenvalue of $P_{+}\left(\epsilon_{1}-\epsilon_{2}, B_{+}\right)$is

$$
\Pi_{N}\left(\epsilon_{1}-\epsilon_{2}, B_{+}\right)=\frac{L_{+}}{\ell} \int_{0}^{\infty} d x L_{N}\left(x^{2}\right) \exp \left[-\frac{x^{2}}{2}-\frac{L_{+}}{\ell} x\left(1-i \frac{\epsilon_{1}-\epsilon_{2}}{\hbar} \tau\right)\right]
$$

and each eigenvalue is degenerate $\left(L^{2} / \pi L_{+}^{2}\right)$ times. $L_{N}$ is the Laguerre polynomial $(N=0,1, \ldots)$ and $L_{+}^{2}=2 \hbar c / e B_{+}$. Thus, the contribution of $P_{+}$to the variance of susceptibility, Eq. (16), is:

$$
\left.\left\langle\Delta \chi^{2}\right\rangle_{+}=\frac{2 s^{2} T^{2}}{L^{4}} \lim _{\substack{B_{1} \rightarrow B \\ B_{2} \rightarrow B}} \frac{\partial^{4} B_{1}^{2} \partial^{2} B_{2}}{\pi L_{+}^{2}} R e \sum_{p, q=0}^{\infty} \sum_{n=3}^{\infty} \sum_{N=0}^{\infty} \frac{L^{2}}{n} \Pi_{N}^{n}\left(\epsilon_{p}+\epsilon_{q}, B_{+}\right)\right](18
$$

where

$\Pi_{N}\left(\epsilon_{p}+\epsilon_{q}, B_{+}\right)=\frac{L_{+}}{\ell} \int_{0}^{\infty} d x L_{N}\left(x^{2}\right) \exp \left\{-\frac{x^{2}}{2}-\frac{L_{+}}{\ell} x\left[1+\frac{2 \pi T \tau}{\hbar}(p+q+1)\right]\right\}$

To obtain the contribution $\left\langle\Delta \chi^{2}\right\rangle_{-}$, corresponding to $P_{-}$, one has to replace $B_{+}$by $B_{-}$, in the argument of $\Pi_{N}$ and in the definition of $L_{+}$. Thus, for any value of the actual magnetic field $B$ (satisfying, of course, $\omega_{c} \tau \ll 1$ ), $\left\langle\Delta \chi^{2}\right\rangle_{-}$ is given by the weak field limit of $\left\langle\Delta \chi^{2}\right\rangle_{+}$.

For $L_{B}, L_{T} \gg \ell$ (the diffusion regime), the integral in (18) is dominated by small $x$, so that $L_{N}\left(x^{2}\right) \simeq 1-N x^{2}$, and

$$
\Pi_{N}^{(\mathrm{dif})} \approx 1-\frac{2 \ell^{2}}{L_{+}^{2}}\left(N+\frac{1}{2}\right)-\frac{2 \pi T \tau}{\hbar}(p+q+1)
$$

which holds for $N \ll\left(L_{+} / \ell\right)^{2}$ and $p, q \ll \hbar / T \tau$. Under these conditions the sum over $n$ in Eq. (18) can be approximated by $-\ln \left(1-\Pi_{N}\right)$ and the expression for the typical value $\left\langle\Delta \chi^{2}\right\rangle^{\frac{1}{2}} \equiv \delta \chi$ can be reduced to the one given in [5]. In the limit of weak field

$$
\delta \chi=1.67 \chi_{0} \frac{L_{T}}{L} k_{F} \ell,
$$


where $\chi_{0}=e^{2} / 12 \pi m c^{2}$.

It is known from the previous studies $[15,18-21]$ that calculations based on the diffusion approximation for $\Pi_{N}$ are quantitatively accurate only when the ratio $\ell / L_{B}$ is extremely small. In order to study larger fields or higher temperatures, in particular when $L_{B}$ or $L_{T}$ become smaller than $\ell$ (the ballistic regime), one must treat eigenvalues $\Pi_{N}$ more accurately. Without resorting to numerics, one can use the approximate expression [20]

$$
\Pi_{N}=\left[4 \frac{\ell^{2}}{L_{+}^{2}}\left(N+\frac{1}{2}\right)+\left(1+\frac{2 \pi T \tau}{\hbar}(p+q+1)\right)^{2}\right]^{-\frac{1}{2}},
$$

which interpolates between the two regimes. In the ballistic regime, as opposed to the case of diffusion, $\Pi_{N}$ is small so that it is sufficient to keep in Eq. (18) only the term $n=3$. Note that the present approach, while giving the correct asymptotic dependence of $\delta \chi$ on $B$ and $T$, is not capable of producing the correct numerical coefficient in the ballistic regime. The reason is the that the ballistic regime is dominated by three-impurity diagrams, and there are several such diagrams besides the $n=3$ diagram of the diffuson or cooperon series.

Eq. (18), together with the approximate expression (22) for $\Pi_{N}$, enables one to consider a broad range of temperatures and fields. Let us first discuss the case of linear response, i.e. $L_{B}>L_{T}$. In this case, as explained above, $\left\langle\Delta \chi^{2}\right\rangle_{+}=\left\langle\Delta \chi^{2}\right\rangle_{-}$and does not depend on $B$. At low temperatures, $L_{T} \gg \ell$, one is back to the diffusion regime, Eq. (21). At high temperatures $\left(L_{T}<\ell\right.$, i.e. $T>\hbar / \tau)$ one enters the ballistic regime, where $\Pi_{N}$ is small. Keeping 
only the term $n=3$ in Eq. (18), one has to compute the sum

$$
\sum_{N=0}^{\infty}\left[4 b\left(N+\frac{1}{2}\right)+t^{2}\right]^{-\frac{3}{2}}
$$

where $b \equiv\left(\ell / L_{+}\right)^{2}$ and $t \equiv(2 \pi T \tau / \hbar)(p+q+1)$. Although the sum can be computed by the Euler-McLaurin formula, it is instructive to extract the $T$-dependence of $\left\langle\Delta \chi^{2}\right\rangle$ by simple power counting. Since there are four derivatives with respect to $B_{1}, B_{2}$ in Eq. (18), one has to look for terms proportional to $B_{+}^{4}$. One power of $B_{+}$comes from the term $L_{+}^{-2}$, and the other three from the function defined by the sum in Eq. (23). Since this function is of the form $t^{-3} f\left(b / t^{2}\right)$, it is clear that the term proportional to $b^{3}$ will be multiplied by $t^{-9}$. The sum over $p$ and $q$ rapidly converges, so that

$$
\left\langle\Delta \chi^{2}\right\rangle \simeq \frac{T^{2}}{L^{2}} \frac{\partial^{4}}{\partial B^{4}}\left[\left(\frac{2 \pi T \tau}{\hbar}\right)^{-9} \ell^{6}\left(\frac{e B}{\hbar c}\right)^{4}\right]
$$

i.e. $\delta \chi \simeq \chi_{0} k_{F} \ell(\ell / L)\left(\ell_{T} / \ell\right)^{\frac{7}{2}}$, in agreement with the arguments of Sec. 3 .

Consider now the opposite case, $L_{B}<L_{T}$, when $\left\langle\Delta \chi^{2}\right\rangle_{+}$(but not $\left\langle\Delta \chi^{2}\right\rangle_{-}$) begins to depend on $B$. Since $\left\langle\Delta \chi^{2}\right\rangle_{+}$decreases with $B$, it follows that for sufficiently strong fields $\left\langle\Delta \chi^{2}\right\rangle$ will reach half of its low-field value. In the diffusion regime, $\ell<<L_{B}<<L_{T},\left\langle\Delta \chi^{2}\right\rangle$ + decays rather slowly, on a typical scale of $\Delta B \simeq \phi_{0} / L_{T}^{2}$. For stronger fields, $L_{B}<<\ell<<L_{T}$, the ballistic regime is reached. In this regime $\left\langle\Delta \chi^{2}\right\rangle_{+}$becomes negligible, compared to $\left\langle\Delta \chi^{2}\right\rangle_{-}$, and keeps decreasing as $L_{B}^{7}$. Indeed, in this case the sum (23) is of order $b^{-3 / 2}$, as long as $p, q<\sqrt{b}(\hbar / 2 \pi T \tau)$, and the expression in the square brackets in Eq. (18) is estimated as $\left(L^{2} / L_{+}^{2}\right) b^{-3 / 2} b(\hbar / 2 \pi T \tau)^{2} \sim \sqrt{B_{+}}$. After differentiating four times with respect to $B_{+}$, one obtains $\left\langle\Delta \chi^{2}\right\rangle_{+} \simeq$ 
$\chi_{0}^{2}\left(k_{F} \ell\right)^{2}\left(L_{B}^{7} / L^{2} \ell^{5}\right)$. Thus, just on the border of the ballistic regime $\left(L_{B} \simeq \ell\right)$, $\left\langle\Delta \chi^{2}\right\rangle_{+} \simeq \chi_{0}^{2}\left(k_{F} \ell\right)^{2}(\ell / L)^{2}$, i.e. is reduced by a factor $\left(\ell / L_{T}\right)^{2}$ as compared to its low-field value.

Various correlation functions can be studied in a similar way. Consider the functions $\langle(\Delta \chi(B) \Delta \chi(B+\Delta B)\rangle \equiv C(B, \Delta B)$. This function, as was mentioned in the previous Section, differs from $\left\langle\Delta \chi^{2}\right\rangle$ only by the limiting value of $B_{2}$, which is now equal to $B+\Delta B$. Therefore, the "cooperon part", $C_{+}$, is a function of $2 B+\Delta B$, whereas the "diffuson part", $C_{-}$, is a function of $\Delta B$. Both $C_{+}$and $C_{-}$decay with $\Delta B$. In addition, $C_{+}$decays as a function of $B$ and becomes negligible for $L_{B} \ll L_{T}$. Thus, for a fixed temperature $\left(L_{T} \gg \ell\right)$ there should exist aperiodic oscillations of magnetic susceptibility, in an individual (typical) sample, with a characteristic period $\Delta B \simeq \phi_{0} / L_{T}^{2}$ and amplitude $\delta \chi \simeq \chi_{0} k_{F} \ell\left(L_{T} / L\right)$. Since $C_{-}(\Delta B)$ does not depend on $B$, these oscillations will persist into the ballistic regime, $L_{B} \ll \ell$, and are limited only by the condition $\omega_{c} \tau \ll 1$. The possible existence of such oscillations, in the diffusion regime, was already mentioned in [6], although it was not realized that these oscillations do not decay even in the ballistic regime. Similar oscillations should exist, when the temperature or the chemical potential are changed by an amount of order $T$ (or $E_{c}$, if $T<E_{c}$ ). Such a change produces a "new sample", and, thus, a corresponding change in the susceptibility.

\section{Interactions}


Interactions can have a profound effect on orbital magnetism. Long ago Aslamazov and Larkin [24] pointed out the existence of an interaction induced paramagnetism. This phenomenon is similar to the interaction induced diamagnetism in superconductors above the critical temperature. Since, however, in a normal metal the interaction is repulsive, one ends up with a paramagnetic term. In two dimensions this term supercedes Landau diamagnetism of non-interacting electrons.

Static disorder modifies but does not destroy the effect. The combined effect of interactions and disorder was studied in detail in $[25,26]$, where the average susceptibility for an ensemble of disordered samples was calculated (for a semiclassical derivation see [27]). The effect is due to the interaction (in the presence of disorder) between a pair of electrons in the Cooper channel. This interaction, at zero magnetic field, produces a small correction to the single particle density of states. In two dimensions the correction, at the Fermi energy $\mu$, is:

$$
\delta \nu_{c} \simeq \frac{1}{\hbar D} \ln \frac{\ln \left(T_{0} / T\right)}{\ln \left(T_{0} \tau / \hbar\right)},
$$

where $T_{0}=\mu \exp \left(1 / \lambda_{0}\right)$ and $\lambda_{0}$ is the effective electron-electron interaction constant. If $\lambda_{0}$ were much smaller than $1 / \ln (\mu / T)$, one could expand the ratio of the logarithms in $\lambda_{0}$, to obtain the first order result $\delta \nu_{c} \simeq$ $\left(\lambda_{0} / \hbar D\right) \ln (T \tau / \hbar)$. Since, however, the aforementioned condition is violated, one should use the renormalized interaction in the Cooper channel which results in the double logarithm in Eq. (25), with $T_{0}$ roughly equal to $\mu$.

The magnitude of the interaction induced paramagnetic susceptibility (in the linear response regime) can be estimated by using the general relation 
between susceptibility $\chi$ and the value of the effective Bohr magneton $\beta_{\text {eff }}$ for particles in question, namely: $\chi \simeq \beta_{\text {eff }}^{2} \nu$, where $\nu$ is the corresponding density of states. The "particles" (cooperons) have charge $2 \mathrm{e}$ and "mass" $\hbar / 2 D$, so that $\beta_{\text {eff }} \simeq e D / c$ (larger by a factor $k_{F} \ell$ than the electron Bohr magneton $e \hbar / 2 m c)$. The corresponding density of states is the correction $\delta \nu_{c}$, Eq. (25). Thus, the average paramagnetic susceptibility is

$$
\left\langle\chi_{p}\right\rangle \simeq \chi_{0} k_{F} \ell \ln \frac{\ln (\mu / T)}{\ln (\mu \tau / \hbar)} .
$$

Note that a similar estimate could have been made for the typical susceptibility of a mesoscopic sample, in the absence of interaction (Sec. 3). Indeed, multiplying $\beta_{\text {eff }}^{2}$ by the mesoscopic correction to the density of states, $|\delta \nu| \simeq 1 / \hbar D$, gives $\chi \simeq k_{F} \ell \chi_{0}$. Let us emphasize that there is a big difference between the mesoscopic correction $\delta \nu$ and the interaction induced correction $\delta \nu_{c}$. The former is a finite size effect, sensitive to temperature and rapidly oscillating with $\mu$. The latter is a robust, intrinsic property of the interacting system, so that the interaction induced paramagnetism is not at all a mesoscopic effect.

The large paramagnetic susceptibility remains, of course, present also in the mesoscopic samples considered in Sec. 3-5. For $T>E_{c}$ the average susceptibility $\langle\chi\rangle$ is already close to its macroscopic limit, Eq. (26). (For $T \ll E_{c}$ some finite size effects in $\langle\chi\rangle$ show up [4].) Thus, the mesoscopic fluctuations discussed in the present paper will occur on the background of $\left\langle\chi_{p}\right\rangle$. These fluctuations do not seem to be affected by interactions in a significant way, similarly to the case of the universal conductance fluctuations, as discussed 
in [16]. However, the full picture of the mesoscopic magnetism in disordered metals, in the presence of interactions and for arbitrary temperatures and fields, has not yet emerged.

\section{Conclusions}

The theory of mesoscopic effects in orbital magnetism of disordered metals was reviewed and extended to higher temperatures and fields. The effects decay with temperature rather gradually and persist to temperatures much higher than the Thouless energy $E_{c}$. One manifestation of mesoscopic orbital magnetism is aperiodic, sample-specific oscillations in magnetization and susceptibility under changes of various external factors - temperature, chemical potential or external magnetic field. The oscillations persist to quite strong fields, limited only by the condition $\omega_{c} \tau \ll 1$. The amplitude of oscillations in the susceptibility is of order $\chi_{0} k_{F} \ell$ at $T \simeq E_{c}$, and slowly decreases at higher temperatures.

One should distinguish between the average susceptibility, measured on a large ensemble of macroscopically identical samples, and the typical susceptibility measured on an individual sample. I am not aware of any measurements of orbital magnetism in disordered mesoscopic metals. The similar phenomenon of orbital magnetic response to an Aharonov-Bohm flux has been investigated, to some extent, experimentally [28-31]. In particular, measurements on single rings, disordered [29] and quasiballistic [30], were made. Since, at low temperatures and weak fields, the magnetic moment of a ring is about the same as of a singly-connected sample of a comparable size, 
the mesoscopic fluctuations studied in the present paper should be amenable to observation.

Some aspects of the phenomenon of mesoscopic orbital magnetism were not covered in this work. We have not discussed mesoscopic effects for fields $\omega_{c} \tau>1$, i.e. in the regime of the de-Haas-van Alphen oscillations or in the quantum Hall regime. Another omission is the mesoscopic magnetism in three dimensions, where a qualitatively new effect shows up. The susceptibility of an individual sample becomes a tensor $\chi_{\alpha \beta}(\alpha, \beta=x, y, z)$, with non-zero off diagonal components, as opposed to the ensemble averaged susceptibility. This happens because only after averaging over the impurities the sample becomes spatially homogeneous. In an individual sample the direction of magnetization does not coincide with the direction of the magnetic field. For instance, for a sample of a spherical shape, the ratio between $\left\langle\Delta \chi_{x z}^{2}\right)^{\frac{1}{2}}$ and $\left\langle\Delta \chi_{z z}^{2}\right\rangle^{\frac{1}{2}}$, is $1 / \sqrt{3}[5,6]$. It would be of interest to investigate different sample shapes and to extend the treatment beyond linear response.

\section{Acknowledgements}

Part of this work was done during the "Extended Research Workshop on Disorder, Chaos and Interaction in Mesoscopic Systems", in Trieste. I am grateful to the organizers for hospitality and to some of the participants - I. Aleiner, B. Altshuler, V. Ambegaokar, Y. Gefen, A. Larkin, P. Mohanti, A. Zyuzin - for useful discussions and explanations. Illuminating conversations with F. von Oppen, L. Pitaevskii and U. Sivan are gratefully acknowledged. The research was supported by the Fund for Promotion of Research at the 
Technion.

\section{References}

1. Landau L.D. and Lifshitz E.M. 1980 Statistical Physics, 3rd Edition, Part 1 (Pergamon Press).

2. Shapiro B. 1993 Physica A200, 498 (Proc. 4-th International Conference on Frontiers in Condensed Matter Physics); Semiconductors 27, 467 (Proc. IPCMP '92).

3. Fukuyama H. 1989 Jour. Phys. Soc. Japan 58, 47.

4. Oh S., Zyuzin A. Yu and Serota R.A. 1991 Phys. Rev. B44, 8858.

5. Raveh A. and Shapiro B. 1992 Europhys. Lett. 19, 109 (Erratum p. $565)$.

6. Raveh A. 1993 Orbital Magnetic Response of Disordered Conductors, Ph.D. Thesis (Technion, Haifa).

7. von Oppen F. and Riedel E.K. 1993 Phys. Rev. 48, 9170.

8. Argaman N., Imry Y., and Smilansky U. 1993 Phys. Rev. B47, 4440.

9. Gutzwiller M.C. 1990 Chaos in Classical and Quantum Mechanics (Springer, Berlin).

10. Agam O. 1994 J. Phys. I France 4, 697.

11. Bogachek E.N. and Gogadze G.A. 1972 Sov. Phys. JETP 36, 973. 
12. Richter K., Ullmo D., and Jalabert R.A., 1996 Phys. Rep. 276, 1.

13. von Oppen F. 1994 Phys. Rev. B50, 17151.

14. Altshuler B.L., Gefen Y., Imry Y., and Montambaux G. 1993 Phys. Rev. B47, 10335.

15. Dyakonov M.I. 1994 Sol. St. Communications 92, 711.

16. Lee P.A., Stone A.D., and Fukuyama H. 1987 Phys. Rev. B35, 1039.

17. Altshuler B.L. and Shklovskii B.I. 1986 Sov. Phys. JETP 64, 127.

18. Altland A. and Gefen Y. 1995 Phys. Rev. B51, 10671.

19. Gasparian V.M. and Zyuzin A.Yu. 1985 Sov. Phys. Solid State 27, 999.

20. Kawabata A. 1984 J. Phys. Soc. Japan 53, 3540.

21. Cassam-Chenai A. and Shapiro B. 1994 J. Phys. I France 4, 1527.

22. Lee P.A. and Payne M.G. 1972 Phys. Rev. B5, 923.

23. Kurkijarvi J., Ambegaokar V. and Eilenberger G. 1972 Phys. Rev. B5, 868.

24. Aslamazov L.G. and Larkin A.I. 1974 Sov. Phys. JETP 40, 321.

25. Altshuler B.L., Aronov A.G. and Zyuzin A.Yu. 1983 Sov. Phys. JETP $57,889$. 
26. Altshuler B.L. and Aronov A.G. 1985 in Electron-Electron Interactions in Disordered Systems, ed. Efros A.L. and Pollak M. (North-Holland).

27. Ullmo D., Richter K., Baranger H.U., von Oppen F., and Jalabert R.A. 1997 Physica E1, 268.

28. Levy L.P., Dolan G., Dunsmuir J., and Bouchiat H. 1990 Phys. Rev. Lett. 64, 2074.

29. Chandrasekhar V., Webb R.A., Brady M.J., Ketchen M.B., Gallagher W.J., and Kleinsasser A. 1991, Phys. Rev. Lett. 67, 3578 (1991).

30. Mailly D., Chapelier C., and Benoit A., 1993 Phys. Rev. Lett. 70, 2020.

31. Mohanty P., Jariwala E.M.Q., Ketchen M.B. and Webb R.A., preprint 